\title{
GENERALIZED STATISTICAL MODELS OF VOIDS AND HIERARCHICAL STRUCTURE IN COSMOLOGY
}

Aram Z. MEKJIAN

Department of Physics and Astronomy, Rutgers University, 136 Frelinghuysen Road, Piscataway, NJ 08854; and Kellogg Radiation Laboratory, California Institute of Technology, 1200 East California Boulevard, Pasadena, CA 91125

Received 2006 June 8; accepted 2006 August 1

\begin{abstract}
Generalized statistical models of voids and hierarchical structure in cosmology are developed. The often quoted negative binomial model and the frequently used thermodynamic model are shown to be special cases of a more general distribution that contains a parameter $a$. This parameter is related to the Lévy index $\alpha$ and the Fisher critical exponent $\tau$, the latter of which describes the power-law falloff of clumps of matter around a phase transition. The parameter $a$, exponent $\tau$, or index $\alpha$ can be obtained from properties of a void scaling function. A stochastic probability variable $p$ is introduced into a statistical model, which represents the adhesive growth of galaxy structure. The galaxy count distribution decays exponentially quickly with size for $p<\frac{1}{2}$. For $p>\frac{1}{2}$, adhesive growth can go on indefinitely, thereby forming an infinite supercluster. At $p=\frac{1}{2}$, a scale-free power-law distribution for the galaxy count distribution is present. The stochastic description also leads to consequences that have some parallels with cosmic string results, percolation theory, and phase transitions.
\end{abstract}

Subject headings: cosmology: theory — large-scale structure of universe

\section{INTRODUCTION}

The distribution of galaxies on large scales shows geometric features such as walls, filaments, and voids. Understanding these features and related issues of clustering and density fluctuations in a gravitational many-body system is a major endeavor in cosmology. Theories of the origins of large-scale structure and anisotropy of the cosmic microwave background have proceeded along several lines. One approach is based on an amplification of quantum fluctuations during inflation. The other main approach is based on symmetry breaking during a phase transition in the early universe, which plays an important role in its evolution in time. Associated with this phase transition are topological defects such as cosmic strings, domain walls, and texture. Loops of cosmic string may be centers for the accretion of galactic matter and be a source of the clumping seen in the distribution of matter. The clustering of galaxies depends on many features that involve the cosmological parameters, the dark matter distribution, and the coupling and evolution of the dark matter component with the luminous component. Another, less dynamical and more phenomenological approach has involved studies of the properties of the distribution of galaxies and the large-scale structure through correlation studies. In this approach, the analysis focuses on the nature of the correlations, looking for patterns that may give physical insight into the processes that are responsible for the structure. One pattern is that of a hierarchical structure. Early methods of analysis of clustering of galaxies were based on two-point correlation functions (Peebles 1980), which offered a lowest-order correlation approach to such clustering. A more recent investigation of the importance of the two-point correlation function at large scales has been carried out by Durrer et al. (2003). Methods for studying correlations of higher order have also been developed. The simplest assumption is that higher order correlations obey a hierarchy of scaling in which higher order correlations are related to lower order two-body correlations. Hierarchical correlations were shown to appear in nonlinear regimes by Peebles (1980). They are commonly discussed by many others, and a recent review with references is given by
Bernardeau et al. (2002). The void probability function (VPF) offers a useful way of understanding some of the higher order correlation features. Recent observations from the $2 \mathrm{dF}$ Galaxy Redshift Survey by Croton et al. (2004) show that a reduced void measure is a useful way of investigating the large-scale structure seen and that the results are in excellent agreement with a paradigm of hierarchical scaling. The physical properties of voids have also been studied by Hoyle \& Vogeley (2004).

Distributions taken from quantum optics and photon count models (Carruthers \& Shih 1983) have been somewhat successfully applied to the analysis of the large-scale structure that is seen. One of the most familiar of these distributions is based on a negative binomial probability description. The negative binomial model implies a specific hierarchical structure to the distribution, which is discussed below. The negative binomial distribution has also been used in particle physics phenomenology and, in particular, in understanding intermittency phenomena. The recent analysis of cosmological data by Croton et al. (2004) has revealed small departures from the negative-binomial result for the VPF. The VPFs associated with other distributions were also considered by them. These included a Gaussian model, a thermodynamic model, a lognormal distribution, and a distribution based on a BBGKY distribution. The negative binomial best characterized the data among the specific distributions considered.

In previous papers (Mekjian 2001; Lee \& Mekjian 2004) and in a different context (particle and nuclear physics, count probabilities in quantum optics), a generalization of the negative binomial model was developed using a theoretical framework based on statistical mechanics. This generalization involved the introduction of a parameter $a$ into a statistical model of count probabilities and nuclear and particle cluster distributions. The formation of clusters in an expanding many-body system of hadronic matter has some aspects that are similar to the clustering of galaxies in an expanding universe mentioned above. The statistical model also has some similarities to droplet models of phase transitions around a critical point. The parameter $a$ is analogous to the Fisher critical exponent $\tau$ (Stanley 1971), which describes the power-law falloff of droplet sizes associated 
with the phenomena of critical opalescence occurring at the critical point of a second-order phase transition. The parameter $a$ can also be related to the Lévy index $\alpha$ (Lévy 1954; Feller 1971; Sato 1999). The importance of the Lévy distribution has been stressed by Mandelbrot (1982). In the context of particle physics and quantum optics, the introduction of the parameter $a$ enables one to connect the negative binomial model $(a=1)$ to a second model $\left(a=\frac{1}{2}\right)$ used in particle physics and in quantum optics and also in descriptions of galaxy clustering. Specifically, in the present context of galaxy clustering, the limiting case of $a=\frac{1}{2}$ has the same void distribution as the thermodynamic model initially due to Saslaw \& Hamilton (1984) and modified by Fry (1986). Fry also considered several other possibilities such as a hierarchical Poisson model and a hierarchical BBGKY model. Moreover, in particle physics the distribution is also connected to a Feynman-Wilson gas (Mekjian 2001), and in quantum optics the count distribution was initially due to Glauber (1963) and was discussed in further detail by Klauder \& Sudarshan (1968). The result arises in photon distributions from field emission from states with Lorentzian line shapes. The negative binomial and this second distribution have somewhat different count probabilities, void scaling, and hierarchical structure properties. Generalizations of the negative binomial distribution in particle phenomenology have also been developed by Hegyi (1993a, 1993b, 1996) and related to the Fox function (Fox 1961).

A review of the importance of various scaling laws in the distribution of galaxies is given by Jones et al. (2005). These authors considered the power-law behavior of the two-point correlation function, both radial and angular, higher order correlation functions such as the three-point correlation function, the hierarchical structure of the $q$ th-order correlations, the power spectrum, counts in cells and the associated void probability function, and fractal and multifractal measures. Scaling features of voids and their connection to fractal models have been studied by Gaite and collaborators (e.g., Gaite \& Manrubia 2002; Gaite 2005, 2006). Studies of the scaling of moments of the counts distribution with cell size $L$ have been proposed as a method of obtaining the scaling dimension of a system (Martínez \& Saar 2002; Borgani 1995). A discussion of the three-point function is given by Takada \& Jain (2003). A recent search for voids is described by Hoyle \& Vogeley (2004). The importance of void statistics was also studied in Balian \& Schaeffer (1989).

\section{CORRELATION FUNCTIONS, HIERARCHICAL STRUCTURE, AND VOID PROBABILITY}

The probability of finding $N$ galaxies in a selected volume $V$ randomly placed gives the count probability $P_{N}$. The void probability is the special case $N=0$, and $P_{0}$ is connected to the mean $k$-point correlation function $\xi_{k}$ (White 1979) through the relation

$$
P_{0}=\exp \left[-\sum_{k=1}^{\infty} \frac{(-\langle N\rangle)^{k}}{k !} \xi_{k}\right]
$$

where $\langle N\rangle$ is the mean number of galaxies in $V$. To obtain $P_{0}$ from $\xi_{k}$, all correlation moments $\xi_{k}$ have to be determined. The relation simplifies in hierarchical models, where the $k$-point correlation function can be expressed in terms of the two-point correlation function through the connection

$$
\xi_{k}=A_{k} \xi_{2}^{k-1}
$$

Such an organization in $\xi_{k}$ is referred to as hierarchical structure. The $A_{k}$ are scaling coefficients determined by the hier- archical model. Using this relation, the term $-\langle N\rangle k \xi_{k}$ in the expression for the void probability simplifies to $\langle N\rangle\left(\langle N\rangle \xi_{2}\right)^{k-1}$. The quantity $\chi \equiv-\ln \left(P_{0}\right) /\langle N\rangle$, called the reduced void probability function, now has the feature of being a function of the specific combination $\langle N\rangle \xi_{2}$ only, namely,

$$
\chi=\sum_{k=1}^{\infty} \frac{A_{k}}{k !}\left(\langle N\rangle \xi_{2}\right)^{k-1}
$$

The behavior of $\chi$ with $\langle N\rangle \xi_{2}$ depends on the scaling amplitudes $A_{k}$. For example, if $A_{k}=(k-1)$ !, the sum over $k$ is simply

$$
\chi=\sum_{k=1}^{\infty} \frac{1}{k}\left(\langle N\rangle \xi_{2}\right)^{k-1}=\frac{1}{\langle N\rangle \xi_{2}} \ln \left(1+\langle N\rangle \xi_{2}\right) .
$$

This particular behavior of $\chi$ as a function of $\langle N\rangle \xi_{2}$ arises from a negative binomial distribution, as discussed in the next section. The $\xi_{2}$ and the fluctuation are related by $\left\langle N^{2}\right\rangle-$ $\langle N\rangle^{2}=\langle N\rangle+\xi_{2}\langle N\rangle^{2}$ or through the factorial moment result $\langle N(N-1)\rangle=\langle N\rangle^{2}\left(1+\xi_{2}\right)$.

Hierarchical pictures have been usefully applied in understanding large-scale structure from a dynamical or evolutionary point of view. Several descriptions have been developed based on hierarchical scaling. For example, small-scale clumps are formed in regions where the density fluctuations are large, and they are produced earlier than large-scale clumps. The smallscale clumps are subsequently captured by large-scale clumps in a hierarchical clustering process. The Press-Schechter theory is a statistical picture of this clustering (Press \& Schechter 1974).

In the present paper, a theoretical framework is developed to describe the large-scale structure in terms of a statistical model based on a canonical and a grand canonical ensemble. The probability $P_{N}$ is obtained from a fugacity expansion of the grand canonical ensemble. Specific choices of the form of the grand canonical ensemble lead to particular probability distributions, such as the negative binomial model and the thermodynamic model. A generalized choice based on a hypergeometric description $(\S 4.8)$ contains these two frequently used cases as special limits. Sections 4.4, 4.5, and 4.6 discuss specific distributions. The void scaling and hierarchical structure of the hypergeometric description are developed in further detail in $\S \S 5.1$ and 5.2. Before presenting these results, a brief summary of some of the ideas to be developed is presented in the next section, using the negative-binomial results to establish some useful quantities to be considered. Further highlights of the results are as follows: Section 4.3 contains a description in terms of clan variables, while $\S 4.7$ introduces a stochastic probability variable $p$ into the theoretical framework. This $p$ can be viewed as an evolutionary variable describing the adhesive growth of galaxy clusters. Parallels and consequences of this picture are further discussed in $\S \S 6$ and 7. Section 6 outlines a formal parallel with a result from cosmic strings, and $\S 7$ discusses of a parallel with percolation theory and phase transitions.

\section{COUNT DISTRIBUTIONS AND PARALLELS WITH QUANTUM OPTICS}

Probability count distributions appear in many area of physics. Of relevance to this paper is a count distribution taken from quantum optics that was subsequently used as a phenomenological model of galaxy count distributions. Specifically, Carruthers 
\& Shih (1983) used the negative binomial distribution for this purpose. The negative binomial probability distribution is

$$
\begin{aligned}
P_{N} & =\left(\begin{array}{c}
N+\kappa-1 \\
N
\end{array}\right) p^{\kappa}(1-p)^{N} \\
& =\left(\begin{array}{c}
N+\kappa-1 \\
N
\end{array}\right)\left(\frac{\langle N\rangle / \kappa}{1+\langle N\rangle / \kappa}\right)^{N}\left(\frac{1}{1+\langle N\rangle / \kappa}\right)^{\kappa} ;
\end{aligned}
$$

the probability $p$ determines the mean number $\langle N\rangle$, and $\kappa$ is a parameter that appears in the expression for the fluctuation $\delta N^{2} \equiv\left\langle N^{2}\right\rangle-\langle N\rangle^{2}$. Namely,

$$
\begin{gathered}
p=\frac{1}{1+\langle N\rangle / \kappa}, \quad\langle N\rangle=\kappa \frac{1-p}{p}, \\
\left\langle N^{2}\right\rangle-\langle N\rangle^{2}=\langle N\rangle(1+\langle N\rangle / \kappa)=\kappa(1-p) / p^{2} .
\end{gathered}
$$

Properties of the negative binomial can also be written in terms of the two variables $\hat{P}$ and $\hat{Q}$ defined by $\hat{Q} \equiv 1 / p$ and $1-p \equiv$ $\hat{P} / \hat{Q}$ or $\hat{P}=(1-p) / p$. Then $\langle N\rangle=\kappa \hat{P}$ and $\delta N^{2}=\kappa \hat{P} \hat{Q}$. Clan variables, introduced by Van Hove (1989), have also been used to characterize the negative binomial and other probability distributions. The clan variable representation for the negative binomial distribution and other infinitely divisible probability distributions is given below in $\S 4.3$. The void scaling function for the negative binomial is

$$
\chi_{\mathrm{NB}}=\frac{1}{\xi_{2}\langle N\rangle} \ln \left(1+\xi_{2}\langle N\rangle\right)
$$

The negative binomial result $\chi_{\mathrm{NB}}$ is a special case of a more general void distribution that involves a parameter $a$. The parameter $a$ is related to the Lévy stability index $\alpha$ and the Fisher critical exponent $\tau$ in $\S 4.8$. The distribution to be developed is based on a generalized statistical model that has a void scaling distribution $\chi_{a}$ given by

$$
\chi_{a}=\frac{1}{(1-a) \xi_{2}\langle N\rangle / a}\left[\left(1+\frac{\xi_{2}\langle N\rangle}{a}\right)^{1-a}-1\right] .
$$

The negative-binomial limit is the $a \rightarrow 1$ limit of $\chi_{a}$. An often quoted void distribution is that of a model referred to as the thermodynamic model (Saslaw \& Hamilton 1984; Fry 1986), which has the special value $a=\frac{1}{2}$.

It is useful to compare the negative binomial distribution with another frequently occurring distribution, the count probability of Poisson statistics, which has a probability distribution $P_{N}$ and variance $\delta N^{2}$ given by

$$
P_{N}=\frac{\langle N\rangle^{N}}{N !} \exp (-\langle N\rangle), \quad \delta N^{2} \equiv\langle N\rangle .
$$

Thus, in the Poisson case $\xi_{2}=0$ and consequently the Poisson void scaling function $\chi_{\mathrm{P}}=1$ for all $\xi_{2}\langle N\rangle$. In galaxy count distributions, the Poisson result represents the extreme limit of VPFs, being independent of the scaling variable $\xi_{2}\langle N\rangle$. By comparison, the importance of the Poisson distribution in quantum optics lies in its connection to coherent states. A Poisson distribution represents a coherent signal, while the negative binomial distribution is a chaotic distribution from noise. The negative binomial distribution is a generalization of the Planck distribution, which has index $\kappa=1$. The Planck distribution gives a statistical enhancement in the occupancy of a quantum level arising from BoseEinstein statistics. In phase space, bosons will clump together because of this statistical attraction, leaving underpopulated or voidlike regions in this space. This can be contrasted with a Poisson distribution with a random distribution of points lacking such correlations. Distributions based on combinations of Poisson and negative binomial behavior are also discussed in $\S 4.5$ and offer the possibility of studying the interplay of these two distributions and their effects on void distributions. A discussion of a combination of a Poisson distribution and the thermodynamic model distribution is developed in $\S 7$, where a parallel with percolation theory is also given.

\section{A STATISTICAL MECHANICS APPROACH}

\subsection{General Considerations}

The connection of probability distributions with statistical mechanics can be seen by considering the connection of generating functions for probability distributions and the grand canonical ensemble as a generating function of the canonical ensemble of a fixed number of particles $N$. The generating function for a probability distribution can be written as

$$
\exp \left[\sum_{k=1}^{\infty} C_{k}\left(u^{k}-1\right)\right]=\sum_{N=0}^{\infty} P_{N} u^{N} .
$$

The extra -1 in the exponent of this equation is the normalization factor necessary so that $\sum_{N=0}^{\infty} P_{N}=1$. The exponential factor $\exp \left(\sum_{k=1}^{\infty} C_{k} u^{k}\right) \equiv Z_{\mathrm{gen}}(\boldsymbol{C}, u)=\sum_{N=0}^{\infty} Q_{N} u^{N}$ generates a set of functions $Q_{N}, N=0,1,2, \ldots, \infty$, such that $P_{N}=$ $Q_{N} / Z_{\text {gen }}(\boldsymbol{C}, u=1)$, with the vector $\boldsymbol{C} \equiv\left(C_{1}, C_{2}, \ldots\right)$. In particle multiplicity distributions, the $C_{k}$ are sometimes referred to as combinants (Gyulassy et al. 1978). The combinants for Poisson statistics are $C_{k}=C_{1} \delta_{k, 1}$, so that only $C_{1} \neq 0$, with $C_{1}=\langle N\rangle$. For the negative binomial distribution, the $C_{k}=x t^{k} / k$ have a distribution obtained from a logarithmic expansion of $-x \ln (1-t)$. The values of $x$ and $t$ determine the mean and fluctuation through

$$
\langle N\rangle=x \frac{t}{1-t}, \quad\left\langle N^{2}\right\rangle-\langle N\rangle^{2}=\langle N\rangle\left(1+\frac{\langle N\rangle}{x}\right) .
$$

Comparing this last result with equation (6), the following identifications can be made: $x \leftrightarrow \kappa$ and $t \leftrightarrow 1-p$.

A strong similarity exists between the generating function for $P_{N}$ and a statistical mechanics connection relating the canonical ensemble's partition function $Z_{N}$ and the grand canonical ensemble's partition function $Z_{\mathrm{gc}}$, which reads

$$
Z_{\mathrm{gc}}(\beta, \mu)=\sum_{N=0}^{\infty} Z_{N} u^{N}=\sum_{N=0}^{\infty} Z_{N} \exp (\beta \mu N) .
$$

Here $u=\exp \beta \mu$ is the fugacity, $\mu$ is the chemical potential, and $\beta=1 / T$, where the Boltzmann constant $k_{\mathrm{B}}=1$. The term $Z_{0}=1$ and corresponds to a system with no particles. The $Z_{0}$ is related to the void probability when discussing void scaling in multiplicity distributions. This parallel between the grand canonical partition function and the generating function of a probability distribution is an essential point used in this paper. Instead of using the combinant notation $C_{k}$, the notation $x_{k}$ will be used in 
the statistical model. The probability that a system has $N$ particles is simply the ratio

$$
P_{N}=\frac{Z_{N}}{Z_{\mathrm{gc}}(\beta, \mu)_{\mu \rightarrow 0}}=\frac{Z_{N}}{\sum_{N=0}^{\infty} Z_{N}} .
$$

The void probability $P_{0}$ follows from

$$
P_{0}=\frac{1}{Z_{\mathrm{gc}}(\beta, \mu)_{\mu \rightarrow 0}}=\frac{1}{\sum_{N=0}^{\infty} Z_{N}} .
$$

\subsection{Examples and Motivations from Statistical Mechanics}

Some examples will now be given to establish some of the methods to be used. For example, a system with $Z_{N}=x^{N} / N$ ! has $\sum Z_{N}=\exp x$. A Poisson probability distribution for $P_{N}$ arises, since

$$
P_{N}=\frac{1}{N !} x^{N} \exp (-x)=\frac{1}{N !}\langle N\rangle^{N} \exp (-\langle N\rangle) .
$$

The last equality follows from the result that $x=\langle N\rangle$. An example of a partition function that has the behavior $Z_{N}=x^{N} / N$ ! is that of a nondegenerate ideal gas of particles. The one-particle partition function is $Z_{1}=x=g_{S} \int V d^{3} p \exp \left(-p^{2} / 2 m T\right) / h^{3}=$ $g_{S} V / \lambda_{T}^{3}$. The $Z_{N}=\left(Z_{1}\right)^{N} / N$ ! and the $Z_{N}$ satisfy the one-term recurrence relation $Z_{N}=x Z_{N-1} / N$. Here $V$ is the volume and $g_{S}$ is the spin degeneracy factor $(=2 s+1)$ of the particle. The $\lambda_{T}^{3}$ is the quantum volume given by $\lambda_{T}^{3}=h^{3} /(2 \pi m T)^{3 / 2}=h^{3} /(2 \pi m / \beta)^{3 / 2}$. The analog of $C_{k}$ is now $x_{k}$, and for this case $x_{k}=x \delta_{1, k}$, so that only $x_{1}$ exists. The grand canonical ensemble for this idealized case is $Z_{\mathrm{gc}}(\beta, \mu)=\exp x u=\exp (x \exp \beta \mu)$. The chemical potential $\mu$ of the grand canonical ensemble is obtained by specifying $\langle N\rangle$ for the system: $\mu=T \ln \left(\langle N\rangle \lambda_{T}^{3} / V\right)$. The Poisson distribution is the high-temperature Maxwell-Boltzmann limit of ideal gases. Non-Poissonian distributions arise when the particles are indistinguishable and the system has some degree of degeneracy, as in Bose-Einstein or Fermi-Dirac distributions. Noninteracting ideal Bose-Einstein gases $(\operatorname{spin} 0,1,2, \ldots)$ have $x_{k}=$ $x / k^{5 / 2}+1 / k($ Feynman 1972), while noninteracting ideal FermiDirac gases $\left(\operatorname{spin} \frac{1}{2}, \frac{3}{2}, \ldots\right)$ have $x_{k}=(-1)^{k+1} x / k^{5 / 2}$. These results depend on the number or dimensions $d$ in the system, which here was taken to be 3 . For $d$ dimensions, the factor $x / k^{5 / 2} \rightarrow\left(L^{d} / \lambda_{T}^{d}\right) /$ $k^{1+d / 2}$ with $L$ the length of one side of $V$. The exponent of $k$, which is $1+d / 2$, determines the rate of falloff of $x_{k}$ with $k$. For these specific cases of noninteracting Fermi-Dirac and BoseEinstein gases, the exponent is well known. For interacting systems near a second-order phase transition, critical exponents appear; an exponent that describes the falloff of cluster yields in a liquid-gas phase transition is the Fisher exponent $\tau$ (Stanley 1971).

Once $x_{k}$ is specified, the canonical partition functions can be obtained from a recurrence relation (Chase \& Mekjian 1995) for the cases considered, which involves the sum

$$
Z_{N}=\frac{1}{N} \sum_{N=0}^{N} k x_{k} Z_{N-k}
$$

with $Z_{0}=1$. This result is an extension of the previous noninteracting ideal gas result $Z_{N}=x Z_{N-1} / N$. The ideal gas result follows when $x_{1}=0$ and $x_{k} \neq 0$ for $k=2,3, \ldots$ The $Z_{\mathrm{gc}}$ can be shown to be

$$
Z_{\mathrm{gc}}=\exp \left(\sum_{k=1}^{\infty} x_{k} u^{k}\right)
$$

(Mekjian 1990a, 1990b).
The canonical ensemble $Z_{N}$ for any $x_{k}$ whose grand canonical ensemble is given by equation (17) also has another representation in terms of a weight over a partition of $N$. The weight over all partitions is the main theoretical framework of statistical ensembles. In particular, a partition of $N$ is specified by a vector $\left(n_{1}, n_{2}, \ldots, n_{N}\right) \equiv \boldsymbol{n}$ subject to the constraint $N=\sum k n_{k}$. The $n_{k}$ is the number of groups or clusters of size $k$. A weight $W_{N}(\boldsymbol{n}, \boldsymbol{x})$, where $\boldsymbol{x} \equiv\left(x_{1}, x_{2}, \ldots, x_{N}\right)$, is given to each $\boldsymbol{n}$, which has a structure

$$
W_{N}(\boldsymbol{n}, \boldsymbol{x})=\prod_{k=1}^{N} \frac{x_{k}^{n_{k}}}{n_{k} !}
$$

The $x_{k}$ will contain the underlying physical quantities. Examples of $x_{k}$ will be given shortly. The $n_{k}$ ! are Gibbs factorials. The $Z_{N}$ is determined by summing $W_{N}(\boldsymbol{n}, \boldsymbol{x})$ over all event-by-event histories or possibilities for the vector $\boldsymbol{n}$ :

$$
Z_{N}=\sum_{n} \prod_{k=1}^{N} \frac{x_{k}^{n_{k}}}{n_{k} !}
$$

This particular structure of the weighting of equation (18) leads to the recurrence relation of equation (16) and the form of equation (17) for $Z_{\mathrm{gc}}$. A further significance of the $x_{k}$ can be developed by considering clan variables.

\subsection{Clan Variables}

Clan variables, introduced by Van Hove (1989), have also been used to characterize the negative binomial and other probability distributions. The clan variables $N_{C}$ and $n_{C}$ are defined as follows: The number of clans (in the notation used in this paper) is $N_{C} \equiv \ln \left[Z_{\mathrm{gc}}(\boldsymbol{x}, u=1)\right]=\sum x_{k}$, while the mean number of particles per clan is just the ratio $n_{C} \equiv\langle N\rangle / N_{C}=\sum k x_{k} / \sum x_{k}$. The $N_{C}$ is the zeroth moment of $\boldsymbol{x}$, while the first three moments of $\boldsymbol{x}$ are related to the mean, fluctuation, and skewness of the probability distribution:

$$
\begin{gathered}
N_{C}=\sum_{k=1}^{\infty} x_{k}, \quad n_{C}=\sum_{k=1}^{\infty} k x_{k} / \sum_{k=1}^{\infty} x_{k}, \\
\langle N\rangle=\sum_{k=1}^{\infty} k x_{k}, \quad\left\langle(N-\langle N\rangle)^{m}\right\rangle=\sum_{k=1}^{\infty} k^{m} x_{k} .
\end{gathered}
$$

The last result is only valid for $m=2$ and $m=3$, with the value $m=2$ giving the variances and $m=3$ the skewness. Since $\ln Z_{\mathrm{gc}}=N_{C}=\sum x_{k}$, the $x_{k}$ represent the contribution to the total $N_{C}$ coming from a cluster or clan of size $k$. The clan variables can also be related to the void variables. The connections are

$$
\begin{gathered}
N_{C}=\ln \left[Z_{\mathrm{gc}}(x, u=1)\right]=-\ln P_{0}, \\
\chi \equiv-\ln \left(P_{0}\right) /\langle N\rangle=N_{C} /\langle N\rangle=1 / n_{C} .
\end{gathered}
$$

This last result shows that the void probability and the moments of the $x_{k}$-distribution are connected. In the next three subsections three examples are given, starting with the familiar negative binomial result but now in the framework of a statistical model. 


\subsection{Negative-Binomial Case}

The form of $x_{k}$ that leads to a negative binomial distribution is $x_{k}=x t^{k} / k$, which yields

$$
\begin{aligned}
Z_{\mathrm{gc}} & =(1-t u)^{-x}=1-\left(\begin{array}{c}
-x \\
+1
\end{array}\right) t u+\left(\begin{array}{c}
-x \\
+2
\end{array}\right)(t u)^{2}-\cdots \\
& =1+\left(\begin{array}{c}
x \\
x-1
\end{array}\right) t u+\left(\begin{array}{c}
x+1 \\
x-1
\end{array}\right)(t u)^{2}+\cdots, \\
Z_{N} & =\frac{x(x+1)(x+2) \ldots(x+N-1)}{N !} t^{N} \\
& =\frac{\Gamma(x+N)}{N ! \Gamma(x)} t^{A}=\left(\begin{array}{c}
x+N-1 \\
N
\end{array}\right) t^{N} .
\end{aligned}
$$

The ratio $Z_{N} / Z_{N+1}=N /[(N+x) t] \rightarrow 1 / t$ as $N \rightarrow \infty$ with $x$ fixed. The $P_{N}$ is

$$
P_{N}=\left(\begin{array}{c}
x+N-1 \\
N
\end{array}\right) t^{N}(1-t)^{x}
$$

For the grand canonical ensemble, $\langle N\rangle=x t /(1-t)$ at $u=1$. Solving for $t$ gives $t=(\langle N\rangle / x) /(1+\langle N\rangle / x)$, which, when substituted into equation (23), gives the negative binomial distribution of equation (5). The variance $\delta N^{2}=x t /(1-t)^{2}=\langle N\rangle(1+\langle N\rangle / x)$. The clan variables $N_{C}$ and $n_{C}$ for the negative binomial are

$$
\begin{gathered}
N_{C}=-x \ln (1-t)=x \ln (1+\langle N\rangle / x), \\
n_{C}=-\frac{t}{(1-t) \ln (1-t)}=\frac{\langle N\rangle / x}{\ln (1+\langle N\rangle / x)}=\frac{1}{\chi} .
\end{gathered}
$$

\subsection{Hybrid Model: Signal/Noise or Coherent/Chaotic Model}

A hybrid model, also used in quantum optics (Klauder \& Sudarshan 1968), extrapolates between two limits, one being a Poisson distribution and the other being a negative binomial distribution. In quantum optics, the Poisson distribution arises from a coherent photon source, while the negative binomial characterizes a chaotic source. To simulate this type of behavior, $x_{k}$ is taken as a mixture $x_{k}=y t^{k}+x t^{k} / k$ (Mekjian 2001). The $Z_{\mathrm{gc}}$ and $Z_{N}$ for this $x_{k}$ are

$$
\begin{gathered}
Z_{\mathrm{gc}}=\frac{1}{(1-t)^{x}} \exp \left(y \frac{t}{1-t}\right), \\
Z_{N}=(-1)^{N+1} U(-N+1, x,-y) \frac{t^{N}}{N !}=N y L_{N-1}^{1}(-x) t^{N}
\end{gathered}
$$

with $U(-N+1, x,-y)$ a confluent hypergeometric function and $L_{N-1}^{1}(-x)$ an associated Laguerre polynomial. The values of $y$ and $t$ are given by a coherent signal parameter $S$ and chaotic noise parameter $\mathrm{Nl}$ through the relations

$$
t=\frac{\mathrm{Nl} / x}{1+(\mathrm{Nl} / x)}, \quad y=S \frac{1}{(\mathrm{Nl} / x)[1+(\mathrm{Nl} / x)]} .
$$

Here $\langle N\rangle=S+\mathrm{N} 1$ and

$$
\left\langle N^{2}\right\rangle-\langle N\rangle^{2}=\langle N\rangle+\langle N\rangle^{2} \frac{\mathrm{Nl}}{\mathrm{Nl}+S}\left[1+\frac{S}{1+(\mathrm{Nl} / x)}\right] \frac{1}{x} .
$$

If $S=0$, then $x_{k}=x t^{k} / k$ and a negative binomial distribution is obtained. If $\mathrm{Nl} \rightarrow 0$, then $x_{k} \rightarrow S \delta_{k, 1}$ and only $x_{1} \neq 0$, so that a Poisson distribution results.

\subsection{Thermodynamic Model}

Another choice for $x_{k}$ that leads to simple results and which is important for this paper is

$$
x_{k}=\frac{x}{k}\left(\begin{array}{c}
2 k-1 \\
k-1
\end{array}\right) \frac{t^{k}}{2^{2(k-1)}} .
$$

This case will be referred to as the thermodynamic model, since some of the results developed here are contained in a previous model, initially developed by Saslaw \& Hamilton (1984) and further studied and modified by Fry (1986) in the context of galaxy clustering. The initial Saslaw \& Hamilton result gives a probability for finding $N$ galaxies of

$$
\begin{aligned}
P_{N}(\langle N\rangle, b)= & \frac{\langle N\rangle(1-b)}{N !}[\langle N\rangle(1-b)+N b]^{N-1} \\
& \times \exp [-\langle N\rangle(1-b)-n b] .
\end{aligned}
$$

The $\langle N\rangle=\sum N P_{N}(\langle N\rangle, b)$, while the fluctuation and skewness are given by

$$
\begin{gathered}
\left\langle(N-\langle N\rangle)^{2}\right\rangle=\langle N\rangle /(1-b)^{2}, \\
\left\langle(N-\langle N\rangle)^{3}\right\rangle=\langle N\rangle(1+2 b) /(1-b)^{4} .
\end{gathered}
$$

The fluctuation can then used to obtain the connection $1 /(1-$ $b)^{2}=\left(1+\xi_{2}\langle N\rangle\right)$ and the void scaling property $-\ln \left(P_{0}\right) /\langle N\rangle=$ $1 /\left(1+\langle N\rangle \xi_{2}\right)^{1 / 2}$. Fry (1986) later modified the void distribution $P_{0}(\langle N\rangle, b)=\exp [-\langle N\rangle(1-b)]=\exp \left[-\langle N\rangle /\left(1+\xi_{2}\langle N\rangle\right)^{1 / 2}\right]$ into the distribution $P_{0}(\langle N\rangle, b)=\exp \left(-\xi_{2}^{-1}\left[\left(1+\xi_{2}\langle N\rangle\right)^{1 / 2}-\right.\right.$ 1]). Fry pointed out that the original Saslaw-Hamilton distribution is not a discrete realization of a continuous background number density but, in the continuum limit of large $\langle N\rangle$, it could be processed into one that is related to such a distribution. The approach developed in this paper gives a systematic method for obtaining such probability functions. As an example, the Fry extension of the thermodynamic result follows from the $x_{k}$ given above. The corresponding probability density $P_{N}$ is given below. As mentioned, this result will be shown to be a special case of a more general result that also includes the popular negative binomial model.

As already pointed out, the probability associated with this model for $x_{k}$ also appears in quantum optics, in a model initially developed by Glauber (1963). The photon count probability arises when considering field emission from Lorentzian line shapes. The reformulation of the model in terms of the statistical model discussed here can be found in Mekjian (2001, 2002), where the model is called the Lorentzian-Catalan model or LC model. The Catalan numbers $\mathrm{Cl}_{k} \equiv(1 / k)([2(k-1)] ! /[(k-1) !(k-1) !])$ appear in equation (28). Using Stirling's approximation, the large- $k$ behavior of the Catalan numbers is $\mathrm{Cl}_{k} \sim 2^{2(k-1)} / \sqrt{\pi} k^{3 / 2}$. This combinatoric factor counts the number of diagrams associated with the evolution of a process when stochastic probability variables are included (see $\S 4.7$ ). The associated $Z_{N}$ is

$$
\begin{aligned}
Z_{N} & =(2 x)^{2 N} U(N, 2 N, 4 x) \frac{t^{N}}{N !} \\
& =\frac{(2 x)^{2 N} t^{N}}{N !}\left[\frac{4^{N / 2} \exp (2 x) x^{1 / 2-N}}{\sqrt{\pi}} K_{N-1 / 2}(2 x)\right],
\end{aligned}
$$


and $Z_{\mathrm{gc}}(u)=\exp \left(2 x\left[1-(1-t u)^{1 / 2}\right]\right)$. The $U$ is a confluent hypergeometric function, while $K_{N-1 / 2}$ is a Bessel $K$-function of fractional order $N-\frac{1}{2}$. The ratio

$$
\frac{U(N, 2 N, 4 x) / N !}{U(N+1,2(N+1), 4 x) /(N+1) !} \rightarrow 4 x^{2}
$$

as $N \rightarrow \infty$, from properties of the confluent hypergeometric function. Using this result, the ratio of canonical partition functions scales as

$$
Z_{N} / Z_{N+1} \rightarrow 1 / t
$$

as $N \rightarrow \infty$. This last ratio is independent of $x$. The probability distribution of $N$ is just $P_{N}=Z_{N} / Z_{\mathrm{gc}}(u)_{u \rightarrow 1}$. The $\langle N\rangle$ and $\delta N^{2}$ are

$$
\begin{gathered}
\langle N\rangle=x t / \sqrt{1-t}, \\
\delta N^{2}=\frac{(2-2 t+t) x t}{2(1-t)^{3 / 2}}=\langle N\rangle+\frac{\langle N\rangle^{2}}{2 x \sqrt{1-t}} .
\end{gathered}
$$

Thus $\xi_{2}=1 /\left[2 x(1-t)^{1 / 2}\right]$ and $\xi_{2}$ depends not only on $x$ but also on $t$. The quantity $\xi_{2}\langle N\rangle=t /[2(1-t)]$ is independent of $x$. The clan variables for the thermodynamic model are

$$
N_{C}=2 x(1-\sqrt{1-t}), \quad n_{C}=\frac{t}{2 \sqrt{1-t}(1-\sqrt{1-t})} .
$$

The asymptotic $1 / k^{3 / 2}$ dependence of the thermodynamic model suggests a close correspondence to Bose-Einstein phenomena in one dimension. In $d$ dimensions, the $x_{k}=\left(L^{d} / \lambda_{T}^{d}\right) / k^{1+d / 2}$ as given

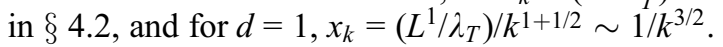

\subsection{Stochastic Variables}

A stochastic probability variable can be incorporated into statistical models. Replacing $x$ and $t$ with

$$
x=\beta_{C} / 4 p, \quad t=4 p(1-p),
$$

the factor $x t^{k} \rightarrow \beta_{C} p^{k-1}(1-p)^{k} 2^{2(k-1)}$. The commonly occurring factors $1-t$ and $(1-t)^{1 / 2}$ are $1-t=(1-2 p)^{2}$ and $(1-2 p)^{1 / 2}=\left[(1-2 p)^{2}\right]^{1 / 2}=|1-2 p|$, with $|1-2 p|$ the absolute value of $1-2 p$; for $p \leq \frac{1}{2},|1-2 p|=1-2 p$, and for $p \geq \frac{1}{2},|1-2 p|=2 p-1$. The quantity $p$ can represent the probability that a cluster of galaxies will grow in size by 1 , while the factor $1-p$ is the probability that this additional growth survives without further increase or accretion. The $p$ is then a gravitational coalescence parameter or adhesion parameter in a statistical approach. Different adhesion approximations have been incorporated into dynamical models, such as in the Zel'dovich (1970) picture. Sahni et al. (1994) studied the dynamical evolution of voids in the framework of an adhesion model.

If this change of variables is incorporated into the $x_{k}$ of equation (28), the following expression for $x_{k}$ results:

$$
x_{k}=\frac{x}{k}\left(\begin{array}{c}
2 k-1 \\
k-1
\end{array}\right) \frac{t^{k}}{2^{2(k-1)}}=\beta_{C} \frac{1}{k}\left(\begin{array}{c}
2 k-1 \\
k-1
\end{array}\right) p^{k-1}(1-p)^{k} .
$$

The Catalan combinatoric factor $\mathrm{Cl}_{k}=(1 / k)([2(k-1)] ! /[(k-$ $\left.1) !]^{2}\right)$ counts the number of diagrams with $k-1$ factors of $p$ and $k$ surviving lines each with probability $1-p$. For $k=1-4$, the Catalan numbers are $\mathrm{Cl}_{1}=1, \mathrm{Cl}_{2}=1, \mathrm{Cl}_{3}=2$, and $\mathrm{Cl}_{4}=5$. The
$\mathrm{Cl}_{1}=1$ represents a single line that survives with probability $1-p$ and has no adhesive junction. Consequently, $x_{1}=1 p^{0}(1-p)^{1}$. The $\mathrm{Cl}_{2}=1$ represents a single diagram with an incoming line that splits into two with probability $p$ at the junction. Each of the two lines that is formed survives with probability $1-p$, giving $x_{2}=1 p(1-p)^{2}$. The $\mathrm{Cl}_{3}=2$ represents the two diagrams that can be obtained from the previous case of $\mathrm{Cl}_{2}=1$ by splitting either line with probability $p$ at the junction. Thus $x_{3}=2 p^{2}(1-$ $p)^{3}$ since two $p$ 's occur, and the three lines that are generated now each survive with probability $1-p$. The $\mathrm{Cl}_{4}=5$ will have five possible diagrams associated with three factors of $p$ and four factors of $1-p$. The $\mathrm{Cl}_{4}=5$ can be generated from $\mathrm{Cl}_{3}=2$ in a manner similar to obtaining $\mathrm{Cl}_{3}=2$ from $\mathrm{Cl}_{2}=1$. The number of diagrams associated with $\mathrm{Cl}_{k}$ grows exponentially fast: $\mathrm{Cl}_{k}=$ $2^{2(k-1)} /\left(k^{3 / 2} \sqrt{\pi}\right)$ for large $k$, using Stirling's approximation. The $Z_{N}$ is

$$
\begin{aligned}
Z_{N} & =\frac{\left[\beta_{C}(1-p)\right]^{N}}{N !} \sqrt{\frac{\beta_{C}}{2 p}} \exp \left(\frac{\beta_{C}}{2 p}\right) K_{N-1 / 2}\left(\beta_{C} / 2 p\right) \sqrt{\frac{2}{\pi}} \\
& =\frac{1}{N !}\left(\frac{\beta_{C}}{p}\right)^{2 N}[p(1-p)]^{N} U\left(N, 2 N,\left(\beta_{C} / p\right)\right),
\end{aligned}
$$

and $Z_{\mathrm{gc}}($ at $u=1)$ is determined by

$$
\begin{aligned}
Z_{\mathrm{gc}} & =\exp \left\{2 \frac{\beta_{C}}{4 p}[1-\sqrt{1-4 p(1-p)}]\right\} \\
& =\exp \left[2 \frac{\beta_{C}}{4 p}(1-|1-2 p|)\right]
\end{aligned}
$$

with

$$
Z_{\mathrm{gc}}= \begin{cases}\exp \beta_{C}, & \text { if } p \leq \frac{1}{2} \\ \exp \left[\beta_{C}(1-p) / p\right], & \text { if } p \geq \frac{1}{2}\end{cases}
$$

The $Z_{\mathrm{gc}}$ is independent of $p$ for $p \leq \frac{1}{2}$ but depends on $p$ for $p \geq \frac{1}{2}$. A further discussion of this point is presented in $\S 8$. The $\langle N\rangle$ and $\delta N^{2}$ are

$$
\langle N\rangle=\beta_{C} \frac{1-p}{|1-2 p|}, \quad \delta N^{2}=\langle N\rangle\left(1+\frac{2 p}{|1-2 p|}\langle N\rangle\right) .
$$

The $\langle N\rangle$ involves $\beta_{C}$ and $p$. The clan variable $N_{C}$, for $p \leq \frac{1}{2}$, is $N_{C}=\beta_{C}=\sum x_{k}$. The $x_{k}$ are the contribution to $N_{C}$ from a clan of size $k$. While each $x_{k}$ is determined by $p$ and $\beta_{C}$, their sum over $k=1,2, \ldots, \infty$ is independent of $p$ for $p \leq \frac{1}{2}$. The mean number of particles per clan $n_{C}=\langle N\rangle / N_{C}=(1-p)|1-2 p|^{-1}$ for $p \leq \frac{1}{2}$. When $p \rightarrow \frac{1}{2},\langle N\rangle \rightarrow \infty$ so $n_{C} \rightarrow \infty$ and $1 / n_{C} \rightarrow 0$. The $x_{k}$ at $p=\frac{1}{2}$ fall as a pure scale-invariant power law, which, for large $k$, is $x_{k} \sim \beta_{C} / 2 \sqrt{\pi} k^{3 / 2}$. Thus, the first moment of $x_{k}$ with respect to $k$ and all higher moments diverge at $p=\frac{1}{2}$ or $t=1$.

\subsection{Generalized Hypergeometric Model: The Parameter a, Lévy Index $\alpha$, and Fisher Exponent $\tau$}

The results of the two previous examples, the binomial and thermodynamic cases, can be united into one model by introducing a variable $a$ into $x_{k}$. Specifically, $x_{k}$ is taken to be of the form

$$
x_{k}=x \frac{[a]_{k-1}}{k !} t^{k}
$$


with $[a]_{m}=a(a+1) \ldots(a+m-1)=\Gamma(a+m) / \Gamma(a)$ and $[a]_{0}=1$. At the special value $a=1$,

$$
x_{k}=\frac{x}{k} t^{k}
$$

which is the negative-binomial result. At $a=\frac{1}{2}$,

$$
x_{k}=\frac{x}{k}\left(\begin{array}{c}
2 k-2 \\
k-1
\end{array}\right) \frac{t^{k}}{2^{2(k-1)}},
$$

which is the thermodynamic model result. For large $k$, the general form of $x_{k}$ varies with $k$ as

$$
x_{k} \sim x \frac{1}{k^{2-a}} t^{k}
$$

This $x_{k}$ can be used in cluster distributions (Lee \& Mekjian 2004), where the Fisher exponent $\tau$ and the parameter $a$ are related by $\tau=2-a$.

For this generalized $x_{k}$ model, the $\langle N\rangle$ and $\delta N^{2}$ are

$$
\begin{gathered}
\langle N\rangle=x t /(1-t)^{a}, \\
\delta N^{2}=\frac{x t[1+(-1+a) t]}{(1-t)^{1+a}}=\langle N\rangle+\frac{a\langle N\rangle^{2}}{x(1-t)^{1-a}} .
\end{gathered}
$$

This last result gives $\xi_{2}=a /\left[x(1-t)^{1-a}\right]$ for the coefficient in front of $\langle N\rangle^{2}$. The choice $a=1$ gives the familiar and simple negative-binomial result $\xi_{2}=1 / x$. The choice $a=\frac{1}{2}$ produces $\xi_{2}=1 /\left[2 x(1-t)^{1 / 2}\right]$, the thermodynamic result.

The logarithm of the grand canonical partition function $Z_{\mathrm{gc}}$ $\left(=\sum x_{k} u^{k}\right)$ is

$$
\begin{aligned}
\ln Z_{\mathrm{gc}} & =(x t u)_{2} F_{1}(a, 1 ; 2 ; t u) \\
& =x\left[1-(1-t)^{1-a}\right] /(1-a)=N_{C},
\end{aligned}
$$

where ${ }_{2} F_{1}(a, 1 ; 2 ; t u)$ is a hypergeometric function. The underlying partition weight $W_{N}(\boldsymbol{n}, \boldsymbol{x})$ is obtained by substituting equation (28) into equation (18). The partition function $Z_{N}$ can be obtained from the recurrence relation of equation (16) using the $x_{k}$ of equation (42).

In general, the hypergeometric function ${ }_{2} F_{1}(a, b ; c ; z)$ is

$$
{ }_{2} F_{1}(a, b ; c ; z)=\sum_{m=0}^{\infty} \frac{[a]_{m}[b]_{m}}{[c]_{m} m !} z^{m} .
$$

The hypergeometric model that was just considered has $b=1$ and $c=2$. More general cases can also be developed by allowing $b$ and $c$ to take other values. The case $b=1$ and $c=2$, with $a$ in the range $0 \leq a \leq 1$, has associated probability distributions that are stable for $t<1$. Probability distribution of his type appear in mathematics as stable Lévy distributions, which have an index $\alpha$ with $1 \geq \alpha \geq 0$. The Lévy index $\alpha$ is connected to $a$, for $1 \geq a \geq 0$, by the simple relationship $1-$ $\alpha=a$. A negative binomial will have $a=1$ and $\alpha=0$, while the case $a=\frac{1}{2}$ will also have $\alpha=\frac{1}{2}$. The Poisson limit is $a \rightarrow 0$ and $\alpha \rightarrow 1$. At $a=0$, only $x_{1}$ is nonzero.

Many cluster distributions and scale-free power laws have $\tau$ in the range $2<\tau<3$. This leads to a faster falloff than $1 / k^{2-a}=1 / k^{1+\alpha}$. The hypergeometric $\operatorname{model}_{2} F_{1}(a, 1 ; 3 ; z)$ with $b=1$ and $c=3$ has $\ln Z_{\mathrm{gc}}=x t u{ }_{2} F_{1}(a, 1 ; 3 ; t u)=\sum x_{k} u^{k}$ with $x_{k} \sim x t^{k} / k^{3-a}$ for large $k$. For $a=\frac{1}{2}$, the $x_{k} \sim x t^{k / k^{5 / 2}}$. Thus, $\sum x_{k}$ and $\sum k x_{k}$ are now both finite. The result for $a=\frac{1}{2}, b=1$, and $c=3$ approximates the behavior of a Bose-Einstein condensate for particles in a three-dimensional volume where $x_{k}=x / k^{5 / 2}$ as given in $\S 4.2$. The $\zeta$-functions that appear in exact theory such as $\zeta(5 / 2)=\sum 1 / k^{5 / 2}=1.341$ and $\zeta(3 / 2)=\sum 1 / k^{3 / 2}=2.612$ are replaced by $4 / 3=1.333$ and $8 / 3=2.667$, respectively. Further discussions of these results will be given elsewhere.

\section{VOID SCALING RELATIONS AND HIERARCHICAL STRUCTURE FUNCTIONS}

\subsection{Void Probability and Void Scaling Relations}

This section contains a more detailed discussion of void scaling relations in $\S 5.1$ and hierarchical structure functions in $\S 5.2$. The void probability is $P_{0}$, and in a statistical model, $P_{0}$ is determined by

$$
P_{0}=Z_{0} / Z_{\mathrm{gc}}(u=1)=1 / Z_{\mathrm{gc}}(u=1)=\exp \left(-N_{C}\right) .
$$

The void function $\chi$ has several useful forms, given by

$$
\chi \equiv-\ln \left(P_{0}\right) /\langle N\rangle=\ln \left(Z_{\mathrm{gc}}\right) /\langle N\rangle=N_{C} /\langle N\rangle=n_{C} .
$$

The scaling behavior of $\chi$ relates $\chi$ to a function associated with the variance written as $\left\langle N^{2}\right\rangle-\langle N\rangle^{2} \equiv\langle N\rangle+\xi_{2}\langle N\rangle^{2}=$ $\langle N\rangle\left(1+\xi_{2}\langle N\rangle\right)$. The quantity of interest is the function $\xi_{2}\langle N\rangle=$ $\left(\left\langle N^{2}\right\rangle-\langle N\rangle^{2}-\langle N\rangle\right) /\langle N\rangle$, which is the ratio of the second factorial moment to the first factorial moment, with the latter being $\langle N\rangle$. The $\xi_{2}\langle N\rangle^{2}$ term gives the departures from Poisson statistics, and $1+\xi_{2}\langle N\rangle$ is an enhancement factor. For a negative binomial distribution, the $\chi$ is

$$
\chi_{\mathrm{NB}}=\frac{1}{\xi_{2}\langle N\rangle} \ln \left(1+\xi_{2}\langle N\rangle\right) .
$$

The corresponding quantity in the thermodynamic model is labeled $\chi_{\mathrm{LC}}$ and is given by

$$
\chi_{\mathrm{LC}}=\frac{\sqrt{1+2 \xi_{2}\langle N\rangle}-1}{\xi_{2}\langle N\rangle}=\frac{2}{\sqrt{1+2 \xi_{2}\langle N\rangle}+1} .
$$

The $\chi_{\mathrm{NB}}$ and $\chi_{\mathrm{LC}}$ are special cases of the hypergeometric model of $\S 4.8$, where $\chi_{a}$ is

$$
\chi_{a}=\frac{1}{(1-a) \xi_{2}\langle N\rangle / a}\left[\left(1+\frac{\xi_{2}\langle N\rangle}{a}\right)^{1-a}-1\right] .
$$

We have $\chi_{\mathrm{NB}}=\chi_{a=1}$ and $\chi_{\mathrm{LC}}=\chi_{a=1 / 2}$. When $\xi_{2}\langle N\rangle \rightarrow \infty$, $\chi_{a} \rightarrow 0$. The vanishing of $\chi_{a}$ with $\xi_{2}\langle N\rangle \rightarrow \infty$ is linked to the behavior $1 / n_{C} \rightarrow 0$ with $t \rightarrow 1$ or $p \rightarrow \frac{1}{2}$. In turn, $1 / n_{C} \rightarrow 0$ is connected to the fact that $\sum k x_{k} \rightarrow \infty$ with $t \rightarrow 1$ or $p \rightarrow \frac{1}{2}$. This result is a consequence of the asymptotic power-law falloff of $x_{k}$ at $t=1$ or $p \rightarrow \frac{1}{2}$, that is, $x_{k} \sim 1 / k^{2-a}=1 / k^{\tau}=1 / k^{1-\alpha}$. For $\sum k x_{k} \rightarrow \infty$, and for $\sum x_{k}$ to be finite, the variable $a$, or Fisher exponent $\tau=2-a$ or Lévy index $\alpha=1-a$, must lie in certain ranges. The exponent $\tau$ is in the range $1<\tau \leq 2$, the index $\alpha$ is in the range $0 \leq \alpha<1$, and the hypergeometric variable $a$ is in the range $0 \leq a<1$.

As shown at the end of $\S 4.8$, generalized hypergeometric models can also be constructed with $x_{k}$ falling off as a higher power of $k$, such as $x_{k} \sim 1 / k^{3-a}=1 / k^{\tau}$. If $3>\tau>2$, then $\langle N\rangle=$ $\sum k x_{k} \sim \sum 1 / k^{\tau-1}$ is finite and $n_{C}=\langle N\rangle / N_{C}$ and $1 / n_{C}$ are finite as well. Then $\chi \rightarrow N_{C} /\langle N\rangle=1 / n_{C}$, a nonzero constant, as 
$\xi_{2}\langle N\rangle \rightarrow \infty$. Thus, the vanishing of $\chi$ with $\xi_{2}\langle N\rangle \rightarrow \infty$ is an indication of $\tau$ lying in the range $1<\tau \leq 2$.

For small $\xi_{2}\langle N\rangle$, equation (53) gives

$$
\chi_{a}=1-\xi_{2}\langle N\rangle / 2
$$

and $\chi_{a}$ is independent of $a$. The $\chi_{a}$ of equation (54) is referred to as the Gaussian approximation. The independence of $\chi_{a}$ from $a$ is a realization of the fact that all models can be replaced by a Gaussian approximation for small $\xi_{2}\langle N\rangle$. The Gaussian approximation can be realized by keeping only terms in $x_{1}$ and $x_{2}$. Then $Z_{\mathrm{gc}}=\exp \left(x_{1}+x_{2}\right),\langle N\rangle=x_{1}+2 x_{2}$, and $\left\langle N^{2}\right\rangle-\langle N\rangle^{2}=$ $x_{1}+4 x_{2}=\langle N\rangle+2 x_{2}=\langle N\rangle+\xi_{2}\langle N\rangle^{2}$. Thus, $2 x_{2}=\xi_{2}\langle N\rangle^{2}$. The void scaling function $\chi_{2} \equiv-\ln \left(P_{0}\right) /\langle N\rangle$ is simply

$$
\chi_{2} \equiv \frac{x_{1}+x_{2}}{\langle N\rangle}=\frac{\langle N\rangle-x_{2}}{\langle N\rangle}=1-\frac{\xi_{2}\langle N\rangle}{2} .
$$

The hybrid coherent signal-chaotic noise model has $\chi_{\mathrm{SNl}}, \xi_{2}$, and $\xi_{2}\langle N\rangle$ given by

$$
\begin{gathered}
\chi_{\mathrm{SN} 1}=\frac{x}{\langle N\rangle} \ln \left(1+\frac{\mathrm{Nl}}{x}\right)+\frac{S}{\langle N\rangle} \frac{1}{[1+(\mathrm{Nl} / x)]}, \\
\xi_{2}\langle N\rangle=\frac{\mathrm{N} 1}{x}\left(1+\frac{S}{\mathrm{~N} 1+S}\right)=\frac{\mathrm{N} 1}{x}\left(1+\frac{S}{\langle N\rangle}\right), \\
\xi_{2}=\frac{1}{x}\left(1-\frac{S}{\langle N\rangle}\right)\left(1+\frac{S}{\langle N\rangle}\right) .
\end{gathered}
$$

For $\mathrm{Nl} \rightarrow 0, \xi_{2} \rightarrow 0$ and $\chi_{\mathrm{SNl}} \rightarrow \chi_{\mathrm{P}}=1$ for any $\xi_{2}\langle N\rangle$, which is the Poisson behavior of $\chi$. For $S \rightarrow 0, \xi_{2} \rightarrow 1 / x$ and the negative-binomial behavior results. For $S \neq 0$ and $\mathrm{Nl} \neq 0$, a void scaling behavior in $\xi_{2}\langle N\rangle$ does not exist. To further illustrate how the void scaling relation is violated by the presence of a Poisson term with strength $S$, the $\chi_{\mathrm{SNl}}$ can be rewritten as

$$
\begin{aligned}
\chi_{\mathrm{SNl}}= & \frac{1-S /\langle N\rangle}{\xi_{2}\langle N\rangle /(1+S /\langle N\rangle)} \ln \left(1+\frac{\xi_{2}\langle N\rangle}{1+S /\langle N\rangle}\right) \\
& +\frac{S}{\langle N\rangle} \frac{1}{1+\xi_{2}\langle N\rangle /(1+S /\langle N\rangle)} .
\end{aligned}
$$

This last relation shows how the negative-binomial scaling is violated by terms involving $S /\langle N\rangle=S /(S+\mathrm{Nl})$, which is the relative level of signal strength to signal plus noise.

\subsection{Hierarchical Structure Relations and Reduced Cumulants}

Another important aspect of the models considered here is their hierarchical structure. The factorial cumulants $f_{q}$ of order $q$ are obtained from an expansion of $\ln Z_{\mathrm{gc}}(\boldsymbol{x}, u)$ :

$$
\ln Z_{\mathrm{gc}}(\boldsymbol{x}, u)=\sum(u-1)^{q} f_{q} / q !=\sum x_{k} u^{k} .
$$

The $f_{q}$ and $x_{k}$ are connected by

$$
\begin{aligned}
f_{q} & =q ! \sum_{n=q}^{\infty}\left(\begin{array}{l}
n \\
q
\end{array}\right) x_{n}=q ! \sum_{n=1}^{\infty}\left(\begin{array}{l}
n \\
q
\end{array}\right) x_{n} \\
& =\sum_{n=1}^{\infty} n(n-1) \ldots(n-q+1) x_{n} .
\end{aligned}
$$

The quantity $f_{2}=2 ! \sum_{n=2} n(n-1) x_{n} / 2=\left\langle A^{2}\right\rangle-\langle A\rangle^{2}-\langle A\rangle$. For a Poisson distribution, $f_{2}=0$ and all higher $f_{q}=0$, since only $x_{1}=0$. For a hypergeometric model with parameter $a$,

$$
f_{q}=\frac{\Gamma(a+q-1)}{\Gamma(a)} \frac{x t^{q-1}}{(1-t)^{a+q-1}} .
$$

The normalized factorial cumulant $k_{q}=f_{q} /\langle N\rangle^{q}$ satisfies a relation connecting $k_{q}$ to $k_{2}$, which reads

$$
k_{q}=A_{q} k_{2}^{q-1} .
$$

The $k_{2}=\xi_{2}$, since $f_{2}=\left\langle N^{2}\right\rangle-\langle N\rangle^{2}-\langle N\rangle=\xi_{2}\langle N\rangle^{2}$. The coefficient $A_{q}$ is given by

$$
A_{q}(a)=\frac{\Gamma(a+q-1)}{\Gamma(a) a^{q-1}} .
$$

The $A_{2}(a)=1$, since $\Gamma(a+1)=a \Gamma(a)$. For the negative binomial, $A_{q}(a=1)=(q-1)$ !. The existence of relations of the form $k_{q}=A_{q} k_{2}^{q-1}$ relating $k_{q}$ to powers of $k_{2}$ expresses a hierarchical structure on a reduced-cumulant level. The above result shows that the hierarchical structure is still preserved for the more general hypergeometric case with variable exponent $a$. The thermodynamic model has $a=\frac{1}{2}$, which when substituted into $A_{q}(a)$ gives $A_{q}\left(\frac{1}{2}\right)=2^{q-1} \Gamma\left(\frac{1}{2}+q-1\right) / \Gamma\left(\frac{1}{2}\right)=(2 q-3)(2 q-$ $5)(2 q-7) \ldots 1$. For $q=3$ (skewness) and $a=\frac{1}{2}$, the $A_{3}\left(\frac{1}{2}\right)=$ $4 \Gamma(5 / 2) / \Gamma\left(\frac{1}{2}\right)=3$. For a negative binomial, $a=1$ and the skewness $A_{3}(1)=2$. The $A_{q}(a)$ increases with decreasing $a$ when $q$ is held constant.

\section{COSMIC STRINGS}

As noted in $\oint 1$, understanding of the origins of large-scale structure and the anisotropy of the cosmic microwave background has proceeded along several lines. One of these approaches is based on symmetry breaking during a phase transition in the early universe, which plays an important role in its evolution in time. Associated with these phase transitions are topological defects such as cosmic strings, domain walls, and texture. Loops of cosmic string may be centers for the accretion of galactic matter and can thus be a source of the clumping of matter seen in the large-scale structure of the universe. However, if strings continually intersect and break into smaller strings, their effect is reduced and, in fact, they might disappear even before they have a chance to accumulate matter that forms galaxies. A simple model was proposed by Smith \& Vilenkin (1987) to study this feature. Here a brief summary is given, since some of its formal features appear in a statistical model when stochastic variables are introduced. Moreover, this formal similarity with results from cosmic strings will be helpful in the next section, which discusses a further parallel with percolation theory.

The Smith-Vilenkin model is based on an initial ancestral string that intersects itself and generates daughter strings in the process of intersection. The probability of intersection is $p$, and the probability of survival without further intersection is $1-p$. Daughter or descendent loops follow the same process with the same probabilities $p$ and $1-p$. The probability of having $n$ loops is

$$
\hat{P}_{n}=A_{n} p^{n-1}(1-p)^{n}
$$

where $A_{n}$ satisfies the recurrence relation $A_{n}=\sum A_{k} A_{n-k}$, where the sum runs from $k=1$ to $k=n-1$ and $A_{1}=1$. This recurrence 
relation follows from the observation that the first daughter of the ancestral loop can produce $k=1,2, \ldots, n-1$ offspring, while the second daughter itself produces $n-k$ descendents. The solution to the recurrence relation is

$$
A_{n}=\frac{1}{n}\left(\begin{array}{c}
2 n-2 \\
n-1
\end{array}\right) .
$$

For large $n$,

$$
\hat{P}_{n}=\frac{1}{n}\left(\begin{array}{c}
2 n-2 \\
n-1
\end{array}\right) p^{n-1}(1-p)^{n} \approx \frac{1}{4 \sqrt{\pi} p} \frac{[4 p(1-p)]^{n}}{n^{3 / 2}} .
$$

For $p \geq \frac{1}{2}$, an infinite cascade occurs, while for $p<\frac{1}{2}$ a finite number of loops are produced. The probability $\mathcal{P}$ that an $\infty$ fragmentation happens satisfies the condition

$$
\mathcal{P}+\sum_{n=1}^{\infty} \hat{P}_{n}=1
$$

and thus $\mathcal{P}=0$ for $0 \leq p \leq \frac{1}{2}$ and $\mathcal{P}=(2 p-1) / p$ when $\frac{1}{2} \leq$ $p \leq 1$. The mean number of loops generated is $\langle n\rangle=(1-p) /$ $(1-2 p)$, and the variance $\delta n^{2}=p(1-p) /(1-2 p)^{3}$. The $\hat{P}_{n}$ have some features that resemble some elements that appear in the thermodynamic model. Specifically, the $x_{k}$ of equation (37) have a similar structure in the stochastic variable $p$ and associated $1-p$ and in the combinatoric factor $A_{n}$. The $x_{k}$ of equation (37) have an additional factor $\beta_{C}$, which is the number of clans associated with the clan variable description. Also, the $\hat{P}_{n}$ start at $n=1$. The probability generated by the $x_{k}$ is, for $0 \leq p \leq \frac{1}{2}$, given by

$$
P_{N}=\frac{1}{N !}\left(\begin{array}{c}
\beta_{C} \\
p
\end{array}\right)^{2 N}[p(1-p)]^{N} U\left(N, 2 N,\left(\beta_{C} / p\right)\right) \exp \beta_{C} .
$$

Comparing the thermodynamic model $P_{N}$ with the cosmic string $\hat{P}_{n}$ for $n=N \gg \beta_{C}$ and for $p \rightarrow \frac{1}{2}^{-}$, the ratio

$$
P_{N} / \hat{P}_{n=N} \rightarrow \beta_{C}
$$

or, for $N \gg \beta_{C}$ and $p \rightarrow \frac{1}{2}^{-}$,

$$
P_{N} \rightarrow \approx \beta_{C} \frac{1}{4 \sqrt{\pi} p} \frac{[4 p(1-p)]^{N}}{N^{3 / 2}} \rightarrow \beta_{C} \frac{1}{2 \sqrt{\pi}} \frac{1}{N^{3 / 2}} .
$$

These results show that the thermodynamic model has a probability distribution for large $N$ and $p \rightarrow \frac{1}{2}$ that is a scaled version of the cosmic string probability, with the scaling factor being the number of clans $\beta_{C}$. The two distributions differ for small $N$. For $\beta_{C}=1$, the two distributions are asymptotically the same, but they still differ for small $N$ because of the void probability $P_{0}$. The $P_{N}<\hat{P}_{n=N}$ for $\beta_{C}=1$ and $p=\frac{1}{2}$. The quantity $P_{N}$ falls as a pure power law $1 / N^{3 / 2}$ and is thus scale-free as $p \rightarrow \frac{1}{2}$. For $p<\frac{1}{2}$, a decreasing exponential term in $N$ is present in $P_{N}^{2}$, that is, the term involving $p$ as $[4 p(1-p)]^{N}=\exp \left(-\left|\lambda_{p}\right| N\right)$ with $\lambda_{p}=\ln [4 p(1-p)]<0$ for $p \neq \frac{1}{2}$. The $P_{N}$ are no longer scalefree for $p \neq \frac{1}{2}$. At $p=\frac{1}{2}$, the $\langle N\rangle \stackrel{2}{\rightarrow} \infty$, since $P_{N} \rightarrow 1 / N^{3 / 2}$. The $\exp \left(-\left|\lambda_{p}\right| N\right)$ term stabilizes the distribution for $p \neq \frac{1}{2}$. An infinite $\langle N\rangle$ is characteristic of a Lévy-unstable distribution. An unstable Lévy distribution can also be made stable by truncating $P_{N}: P_{N}=0$, for $N>N_{C}$, where $N_{C}$ is a cut off $N$.

\section{PARALLELS WITH PERCOLATION THEORY AND PHASE TRANSITIONS}

The region $p>\frac{1}{2}$ in the thermodynamic model with stochastic variables corresponds to a new branch where the number of clans changes from $N_{C}=\beta_{C}$, a constant for all $p \leq \frac{1}{2}$, to a $p$-dependent quantity. This sudden change suggests an Ansatz in which an additional quantity is introduced to keep $N_{C}$ a constant. This section explores this possibility and its consequences. Further investigations are in progress, and the results presented here are somewhat tentative. First, some motivation is given for this possibility. In percolation Stauffer \& Aharony (1994), when the bond or site probability crosses the critical $p_{\text {crit }}$, an infinite cluster appears with strength $S$ that depends on $p$. For percolation on a Bethe lattice or Cayley tree, this $S=p\left(1-[(1-p) / p]^{3}\right)$. In Bose-Einstein condensation, when the temperature falls below $T_{\text {crit }}$ the ground-state occupancy is singled out and treated separately. Then, suddenly, at $T_{\text {crit }}$ the ground state starts to be filled with a macroscopic number of particles. In the cosmic-string discussion given above, for $p>\frac{1}{2}$ a $\mathcal{P}$ suddenly appeared that represented the fact that the splitting of the ancestral loop and descendents now goes on forever. The $\mathcal{P}=(2 p-1) / p$. The extra amount needed to keep the clan number fixed at $N_{C}=\beta_{C}$ for $p>\frac{1}{2}$ is labeled $\varphi_{C}$, and this $\varphi_{C}=\Theta\left(p-\frac{1}{2}\right) \beta_{C}(2 p-1) / p$, where $\Theta\left(p-\frac{1}{2}\right)=1$ for $p>\frac{1}{2}$ and $\Theta\left(p-\frac{1}{2}\right)=0$ for $p<\frac{1}{2}$. The additional $\varphi_{C}$ is part of the $\sum x_{k}=N_{C}$, such that the sum is now fixed at the constant $\beta_{C}$ for all $0 \leq p \leq 1$. The $\varphi_{C}$ is only defined by its total sum rule and not by a specific distribution in $x_{k}$. To proceed, a modified form of a signal/noise or Poisson/negative binomial model ( $\S 4.5)$ is used as a possible option. The Poisson signal level will be $\varphi_{C}$, and the thermodynamic model will now replace the negative binomial. The $\varphi_{C}$ is then an additional Poisson piece, and the total $\varphi_{C}$ strength is added to $x_{1}$ such that $x_{1} \rightarrow(1-p)+\varphi_{C}=$ $(1-p)+\Theta\left(p-\frac{1}{2}\right) \beta_{C}(2 p-1) / p$. The $x_{k}$, for $k \neq 1$, are unchanged and therefore $x_{k}=\mathrm{Cl}_{k} p^{k-1}(1-p)^{k}$. For $p \leq \frac{1}{2}$, the additional Poisson amplitude vanishes, while for $p \rightarrow 1$ only a pure Poisson distribution survives. The $\langle N\rangle$ is now

$$
\langle N\rangle=\varphi_{C}+\beta_{C} \frac{1-p}{|2 p-1|},
$$

and

$$
\left\langle N^{2}\right\rangle-\langle N\rangle^{2}=\varphi_{C}+\beta_{C} \frac{(1-p)\left(1-2 p+2 p^{2}\right)}{|2 p-1|^{3}} .
$$

Thus, as before, for $p \leq \frac{1}{2}$ the additional Poisson term vanishes and

$$
\begin{aligned}
\chi=1-\frac{p}{1-p} & =\frac{1}{\xi_{2}\langle N\rangle}\left(\sqrt{1+2 \xi_{2}\langle N\rangle}-1\right) \\
& =\frac{2}{\sqrt{1+2 \xi_{2}\langle N\rangle}+1},
\end{aligned}
$$

where $\xi_{2}=2 p /\left[\beta_{C}(1-2 p)\right]$ and $\xi_{2}\langle N\rangle=2 p(1-p) /(1-2 p)^{2}$. The $p$ in terms of $r \equiv 2 \xi_{2}\langle N\rangle$ is $p=\frac{1}{2}-1 /\left[2(1+r)^{1 / 2}\right]$, and $p /(1-p)=\left[(1+r)^{1 / 2}-1\right] /\left[(1+r)^{1 / 2}+1\right]$. However, for $p>\frac{1}{2}$ a scaling relation for $\chi$ in terms of the scaling variable $\xi_{2}\langle N\rangle$ is no longer valid, because of the presence of the Poisson part $\varphi_{C}$. The $\chi$ now has a contribution from $\varphi_{C}$ and reads $\left(p>\frac{1}{2}\right)$

$$
\chi=\frac{\beta_{C}}{\varphi_{C}+\beta_{C}(1-p) /|2 p-1|}=\frac{1}{\hat{\varphi}_{C}+(1-p) /|2 p-1|},
$$


where $\varphi_{C}=\beta_{C} \hat{\varphi}_{C}$. The count distribution follows from the recurrence relation of equation (16).

\section{CONCLUSIONS AND SUMMARY}

A statistical approach has been introduced that can be used to discuss various features associated with the distribution of galaxies and the large-scale structure of the universe. These features include the clumping of galaxies, the associated production of voids, and the hierarchical structure of correlations. Within the statistical framework, a generalized model was considered that contains a continuous parameter or variable called $a$. This parameter is related to the Lévy stability index $\alpha$ in probability distributions $(1-a=\alpha)$ and the Fisher critical exponent $\tau$ in cluster phenomena $(\tau=2-a)$. The Fisher index $\tau$ describes the power-law falloff of cluster sizes or clumps of matter at a phase transition or critical opalescence point. The Lévy index characterizes non-Gaussian probability distributions that have power-law behavior. The continuous parameter $a$, exponent $\tau$, or index $\alpha$ can be obtained from properties of a void scaling function. Namely, the value of $a, \alpha$, or $\tau$ governs the asymptotic behavior of $\chi$ in terms of the scaling variable $\xi_{2}\langle N\rangle$. Moreover, a $\chi$ that vanishes with $\xi_{2}\langle N\rangle$ was shown to exclude values $\tau>2$. For $\tau>2, \chi$ approaches a nonzero value as $\xi_{2}\langle N\rangle \rightarrow \infty$. Special values of $a$ produce the void and hierarchical structures of frequently used phenomenological models for galaxy count distributions in cosmology. Specifically, the value $a=1$ gives the negative binomial distribution, while $a=\frac{1}{2}$ gives the distribution from the thermodynamic model. As $a \rightarrow 0$, a random uncorrelated Poisson limit is realized in the galaxy count distribution. Results based on combinations of Poisson plus chaotic distributions, such as the negative binomial distribution, were also considered. How the Poisson part affects the hierarchical scaling behavior associated with the negative binomial part was studied in detail. Parallels were drawn with Bose-Einstein statistical correlations, which lead to clumping of photons in phase space. These parallels with Bose-Einstein phenomena were also used to motivate the form of the statistical model that was developed. The Van Hove (1989) clan description of clumps and voids was incorporated into the description and connected to features of the statistical model.

A formulation of the statistical model in terms of stochastic probability variables was also given, namely, a probability $p$ was incorporated into the statistical picture, where $p$ is the probability that a galaxy adheres to another galaxy or group of galaxies to form a larger cluster of galaxies. A factor $1-p$ in the description represents the probability that a cluster of galaxies survives without further adhesive processes. Thus, an evolutionary growth process is incorporated into this phenomenological description of galaxy structure. A parallel was drawn with percolation phenomena, where $p$ represents either the bond probability of adjacent points on a lattice or the site probability of a point on the lattice. For percolation on a lattice, when $p \geq p_{\text {crit }}$ an infinite cluster appears, where $p_{\text {crit }}$ is a critical value of $p$. At $p=p_{\text {crit }}$, a pure scale-invariant power law in cluster sizes manifests itself. For a thermodynamic model of galaxy clustering, the behavior of the distribution is also quite different for $p \leq \frac{1}{2}$ and $p>\frac{1}{2}$ and parallels the result from percolation theory. Namely, for $p<\frac{1}{2}$ the adhesion is terminated and the galaxy count distribution decays exponentially quickly with the size of the galaxy cluster. For $p \geq \frac{1}{2}$, the adhesion can go on forever and an infinite supercluster is formed. For $p=\frac{1}{2}$, the probability distribution becomes a scaleinvariant power law. This model also has some formal results that are similar to cosmic-string results, which were briefly mentioned.

This work was supported in part by the Department of Energy under grant DE-FG02-96ER-40987.
Balian, R., \& Schaeffer, R. 1989, A\&A, 220, 1

Bernardeau, F., Colombi, S., Gaztañaga, E., \& Scoccimarro, R. 2002, Phys. Rep., 367, 1

Borgani, S. 1995, Phys. Rep., 251, 1

Carruthers, P., \& Minh, D. V. 1983, Phys. Lett. B, 131, 116

Chase, K. C., \& Mekjian, A. Z. 1995, Phys. Rev. C, 52, 2339

Croton, D. J., et al. 2004, MNRAS, 352, 828

Durrer, R. A., Gabrielli, A., Joyce, M., \& Sylos Labini, F. 2003, ApJ, 585, L1

Feller, W. 1971, An Introduction to Probability Theory and Its Applications, Vol. 2 (2nd ed.; New York: Wiley)

Feynman, R. P. 1972, Statistical Mechanics: A Set of Lectures (Reading, MA: Benjamin/Cummings)

Fox, C. 1961, Trans. Am. Math. Soc., 98, 395

Fry, J. N. 1986, ApJ, 306, 358

Gaite, J. 2005, Europhys. Lett., 71, 332

2006, preprint (astro-ph/0604202)

Gaite, J., \& Manrubia, S. C. 2002, MNRAS, 335, 977

Glauber, R. J. 1963, Phys. Rev. Lett., 10, 84

Gyulassy, M., Kauffman, S. K., \& Wilson, L. 1979, Phys. Rev. C, 20, 2267

Hegyi, S.1993a, Phys. Lett. B, 309, 443

1993b, Phys. Lett. B, 318, 642

1996, Phys. Lett. B, 388, 837

Hoyle, F., \& Vogeley, M. S. 2004, ApJ, 607, 751

Jones, B. J. T., Martínez, V. J., Saar, E., \& Trimble, V. 2005, Rev. Mod. Phys., 76,1211

Klauder, J., \& Sudarshan, E. C. G. 1968, Fundamentals of Quantum Optics (New York: W. A. Benjamin)

\section{REFERENCES}

Lee, S. J., \& Mekjian, A. Z. 2004, Nucl. Phys. A, 730, 514

Lévy, P. 1954, Theorie de l'addition des variables aléatoires (2nd ed.; Paris: Gauthier-Villars)

Mandelbrot, B. B. 1982, The Fractal Geometry of Nature (San Francisco: Freeman)

Martínez, V. J., \& Saar, E. 2002, Statistics of the Galaxy Distribution (Boca Raton: Chapman \& Hall/CRC)

Mekjian, A. Z. 1990a, Phys. Rev. Lett., 64, 2125

1990b, Phys. Rev. C, 41, 2103

2001, Phys. Rev. Lett., 86, 220

2002, Phys. Rev. C, 65, No. 014907

Peebles, P. J. E. 1980, The Large-Scale Structure of the Universe (Princeton: Princeton Univ. Press)

Press, W. H., \& Schechter, P. 1974, ApJ, 187, 425

Sahni, V., Sathyaprakash, B. S., \& Shandarin, S. F. 1994, ApJ, 431, 20

Saslaw, W. C., \& Hamilton, A. J. S. 1984, ApJ, 276, 13

Sato, K. I. 1999, Lévy Processes and Infinitely Divisble Distributions (Cambridge: Cambridge Univ. Press)

Smith, A. G., \& Vilenkin, A. 1987, Phys. Rev. D, 36, 987

Stanley, H. E. 1971, Introduction to Phase Transitions and Critical Phenomena (New York: Oxford Univ. Press)

Stauffer, D., \& Aharony, A. 1994, Introduction to Percolation Theory (rev. 2nd ed.; London: Taylor \& Francis)

Takada, M., \& Jain, B. 2003, MNRAS, 340, 580

Van Hove, L. 1989, Phys. Lett. B, 232, 509

White, S. D. M. 1979, MNRAS, 186, 145

Zel'dovich, Ya. B. 1970, A\&A, 5, 84 